\title{
High level of serum apolipoprotein A-I is a favorable prognostic factor for overall survival in esophageal squamous cell carcinoma
}

Xue-Ping Wang ${ }^{1 \dagger}$, Xiao-Hui Li ${ }^{1 \dagger}$, Lin Zhang ${ }^{1,3}$, Jian-Hua Lin ${ }^{1}$, Hao Huang ${ }^{2}$, Ting Kang ${ }^{1}$, Min-Jie Mao ${ }^{1}$, Hao Chen ${ }^{1 *}$ and Xin Zheng ${ }^{1 *}$

\begin{abstract}
Background: Noninvasive prognostic tools for esophageal squamous cell carcinoma (ESCC) are urgently needed. Serum lipids and lipoproteins are used for the prognosis of certain diseases; however, the prognostic value of serum apolipoprotein A-I (ApoA-I) in ESCC has not been described.

Methods: Pre-treatment serum lipids and lipoprotein concentrations (including ApoA-I, Apo-B, HDL-C, LDL-C, TC and TG) were analyzed retrospectively and compared between 210 patients with ESCC and 219 healthy controls. The prognostic significance of serum lipids and lipoproteins was determined by univariate and multivariate Cox hazard models in ESCC.

Results: Clinical characteristics (age, sex, pT status, pN status, pM status, pTNM status, histological differentiation or alcohol index) had no influence on baseline ApoA-l level. Serum ApoA-I, HDL-C, LDL-C, and TC levels were significantly lower and Apo-B was significantly higher in ESCC patients than in normal controls. On univariate analysis, ApoA-I, alcohol index, pT status, pN status and pTNM status were associated with significantly poor survival, and ApoA-I $(p=0.039)$, alcohol index $(p=0.037)$ and pTNM status $(p=0.000)$ were identified as prognostic factors associated with shorter survival in the multivariate analysis.
\end{abstract}

Conclusions: Overall survival was shorter in ESCC patients with decreased pre-treatment ApoA-l levels. Our findings suggest that serum ApoA-l level should be evaluated as a predictor of survival in patients with ESCC.

Keywords: Apolipoprotein A-I, ESCC, Prognosis, Overall survival

\section{Background}

Esophageal squamous cell carcinoma (ESCC), the predominant histologic type of esophageal cancer, is one of the most lethal malignancies of the digestive system and the sixth leading cause of cancer mortality worldwide [1, 2]. Despite improvements in treatment strategies, including surgical techniques and adjuvant chemoradiation, the overall 5-year survival rate of ESCC patients

\footnotetext{
* Correspondence: chenhao@sysucc.org.cn; zhengxin@sysucc.org.cn †Equal contributors

'Department of Laboratory Medicine, State Key Laboratory of Oncology in South China, Collaborative Innovation Center for Cancer Medicine, Sun Yat-sen University Cancer Center, Guangzhou, Guangdong 510060, People's Republic of China

Full list of author information is available at the end of the article
}

treated with surgery alone is less than $20 \%[3,4]$. In recent years, many serum biomarkers, such as SCC, CYFRA21-1, and CEA, have been used to predict the survival of ESCC patents. However, these markers have limited sensitivity and specificity and are not entirely reliable [5-7]. Therefore, the identification of accurate biomarkers for ESCC is necessary to improve the clinical outcome of patients.

Abnormal levels of lipids have been shown to be closely correlated with tumor progression in several cancers [8,9]. Cholesterol synthesis is enhanced in cancer cells compared with normal cells, as tumor cells need excess cholesterol and cholesterol biosynthesis pathway intermediates to maintain a high level of

\section{$\int$ Biomed Central}


proliferation [10]. Evidence for the activation of lipid metabolism in tumor cells can be provided by quantifying the products of lipid metabolism such as apolipoprotein A-I (ApoA-I) in the serum of cancer patients [11-13].

ApoA-I is a major high-density lipoprotein cholesterol (HDL-C) component in serum, which constitutes approximately $70 \%$ of the apolipoprotein content of HDL-C particles [14]. ApoA-I can function as an enzyme cofactor, receptor ligand, and lipid transfer carrier involved in the regulation of lipoprotein metabolism $[9,15,16]$. Beyond the known functions of ApoA-I, it may indirectly promote tumor survival through the other activation. It is overexpressed in patients with recurrent head and neck squamous cell carcinoma [17]. High ApoA-I concentration is associated with increased risk of cancer, in particular head and neck squamous cell carcinoma [18], whereas the prognostic roles of ApoA-I, Apo-B, HDL-C, low-density lipoprotein cholesterol (LDL-C), total cholesterol (TC), and triglycerides (TG) remain unclear. ApoA-I is a risk and prognostic factor in nasopharyngeal carcinoma [19]; however, its prognostic value remains unclear in ESCC.

To clarify the association between lipid profiles and ESCC, this study was designed to elucidate the associations between alterations of in vivo lipid metabolism and the occurrence of ESCC by measuring the levels of a number of relevant lipids in the pre-therapy serum of patients with ESCC in comparison with those of healthy participants.

\section{Methods}

\section{Patients}

Between January 2007 and July 2009, 210 eligible patients (150 men and 60 women; ages $36-79$ years, median 58 years) diagnosed with ESCC at the Sun Yat-sen University Cancer Center were enrolled into this retrospective study. The demographic details are described in Table 1. All of the patients met the diagnostic criteria for ESCC. Exclusion criteria were as follows: (1) patients treated with medication or taking hormone replacement therapy or any drugs known to affect lipid metabolism before serum collection; (2) patients with concomitant diseases associated with increased serum lipid and lipoprotein levels (i.e., diabetes, hyperlipidemia, or metabolic syndrome); (3) other types of malignancy. Based on medical records, the tumor differentiation grades were classified according to the World Health Organization criteria. Stage was recorded based on the American Joint Committee on Cancer guidelines (2009) [20]. All patients received treatment. Clinical information, including demographic data, pathological tumor node metastasis (pTNM) stage, smoking status, alcohol consumption, and overall survival (OS) data were available for all patients. Tobacco index was calculated as cigarettes per day multiplied by years of smoking, and patients were divided into two groups: smoking and nonsmoking. Alcohol index was assessed as drinking or not drinking. A total of 219 healthy participants (137 men and 82 women; ages 23-79 years, median 54 years) were recruited from the physical examination department at Sun Yat-Sen University Cancer Center.

Prior to use of these serum, informed consent was obtained from each of the patients and healthy participants. All of them provided written informed consent. In our institution, patients were generally followed up every 3 months in the first years, every 6 months for the following 2 years, and annually thereafter for patients without evidence of recurrence. The last follow-up was in October 2015, inform consent and survival status was verified again through direct telecommunication with the patient or their family (performed by The Medical Information Unit in our Cancer Center). This study was approved by the Institute Research Ethics Committee of the Sun Yat-Sen University Cancer Center, Guangzhou, China.

\section{Laboratory measurements}

As part of the physical examination, peripheral blood was collected from the patients between 7 and 8 a.m., before treatment, clotted at room temperature, and centrifuged at $3500 \mathrm{r} / \mathrm{min}$ for $8 \mathrm{~min}$. The levels of ApoA-I, Apo-B, HDL-C, LDL-C, TC, and TG were measured using a Hitachi 7600 automatic biochemical analyzer (Hitachi HighTechnologies, Tokyo, Japan). ApoA-I and Apo-B were measured using immunoturbidimetry; HDL-C and LDL-C were detected by the selective elimination method (direct method) and selective protection method, respectively; serum TC was measured by the CHOD-PAP method and TC was tested using the GPO-PAP method. All reagents used in this study were provided by Wako Pure Chemical Industries, Japan.

\section{Statistical analysis}

All statistical tests were performed with SPSS 16.0 for Windows software (SPSS, Chicago, IL, USA). OS was calculated between the first diagnosis of ESCC and death, or the date of the last follow-up. Data were expressed as the mean and standard deviation (mean $\pm \mathrm{SD}$ ), and the correlation between ApoA-I and clinical characteristics was assessed using the Mann-Whitney $U$ test and $\chi^{2}$ test. The differences between ESCC patients and healthy donors were compared using the unpaired Student's $t$-test. Univariate and multivariate analyses of clinical variables were performed using Cox proportional hazards regression models. The results of this survey were analyzed using the Kaplan-Meier survival curves with the log-rank test and proportional hazard model. The correlation between ApoA-I and alcohol index was analyzed using the Spearman rank correlation test. $P$ values $<0.05$ were regarded 
Table 1 The levels of ApoA-I between the ESCC patients and healthy controls

\begin{tabular}{|c|c|c|c|c|c|c|}
\hline \multirow[t]{2}{*}{ Characteristics } & \multicolumn{2}{|l|}{ ESCC } & \multirow[t]{2}{*}{$p$ Value } & \multicolumn{2}{|l|}{ Controls } & \multirow[t]{2}{*}{$p$ Value } \\
\hline & Numbers & Median (range) & & Numbers & Median (range) & \\
\hline Age, years & & & 0.973 & & & 0.733 \\
\hline$<58$ & 102 & $1.21(0.83-1.64)$ & & 105 & $1.56(0.8-2.32)$ & \\
\hline$\geq 58$ & 108 & $1.21(0.71-2.05)$ & & 114 & $1.59(1.23-2.11)$ & \\
\hline Sex & & & 0.084 & & & 0.887 \\
\hline Male & 150 & $1.16(0.71-2.05)$ & & 137 & $1.56(0.8-2.24)$ & \\
\hline Female & 60 & $1.25(0.81-1.89)$ & & 82 & $1.58(1.05-2.32)$ & \\
\hline pT status & & & 0.175 & & & \\
\hline рT 1 & 17 & $1.15(0.95-1.39)$ & & & & \\
\hline pT 2 & 40 & $1.26(0.88-2.05)$ & & & & \\
\hline pT 3 & 128 & $1.20(0.71-1.84)$ & & & & \\
\hline pT 4 & 25 & $1.12(0.87-1.6)$ & & & & \\
\hline pN status & & & 0.094 & & & \\
\hline pN 0 & 114 & $1.25(0.8-2.05)$ & & & & \\
\hline pN 1 & 96 & $1.16(0.71-1.8)$ & & & & \\
\hline pM status & & & 0.828 & & & \\
\hline pM 0 & 197 & $1.21(0.71-2.05)$ & & & & \\
\hline pM 1 & 13 & $1.16(0.92-1.59)$ & & & & \\
\hline pTNM status & & & 0.226 & & & \\
\hline Stage I & 15 & $1.15(1.03-1.39)$ & & & & \\
\hline Stage II & 103 & $1.25(0.8-2.05)$ & & & & \\
\hline Stage III & 80 & $1.15(0.71-1.8)$ & & & & \\
\hline Stage IV & 12 & $1.22(0.92-1.59)$ & & & & \\
\hline Histological differentiation & & & 0.393 & & & \\
\hline DCIS & 3 & $1.61(1.12-1.67)$ & & & & \\
\hline Low & 66 & $1.22(0.8-1.89)$ & & & & \\
\hline Moderate & 98 & $1.19(0.71-2.05)$ & & & & \\
\hline Well & 43 & $1.17(0.81-1.6)$ & & & & \\
\hline Alcohol index & & & 0.815 & & & \\
\hline Yes & 96 & $1.19(0.71-1.64)$ & & & & \\
\hline No & 114 & $1.21(0.8-2.05)$ & & & & \\
\hline
\end{tabular}

as indicating statistically significant differences. All reported $P$ values are two sided.

\section{Results}

Relationship of ApoA-I level with clinical characteristics

This study was a retrospective review that included 210 ESCC patients between January 2007 and July 2009. At the time of the last follow-up, 115 (54.76 \%) of the 210 patients had died.

The associations between median serum ApoA-I levels and clinical variables in 210 ESCC patients and controls are presented in Table 1 . In the entire cohort, age, sex, pT status, $\mathrm{pN}$ status, $\mathrm{pM}$ status, pTNM status, histological differentiation or alcohol index had no influence on baseline ApoA-I level both in ESCC patients and controls.

\section{Pre-therapy serum levels of lipids in ESCC patients and healthy controls}

The levels of lipids and lipoproteins were compared between ESCC patients and healthy controls to investigate lipid abnormalities associated with ESCC (Table 2). The pre-therapy serum levels of ApoA-I $(1.22 \pm 0.22 \mathrm{mg} / \mathrm{dL})$, HDL-C $(1.22 \pm 0.32 \mathrm{mg} / \mathrm{dL})$, and TC $(4.98 \pm 0.95 \mathrm{mg} / \mathrm{dL})$ in ESCC patients were significantly lower than those in the age and sex matched normal controls (ApoA-I: $1.58 \pm$ $0.24 \mathrm{mg} / \mathrm{dL} ;$ HDL-C: $1.43 \pm 0.33 \mathrm{mg} / \mathrm{dL} ; \mathrm{TC}: 5.61 \pm$ $1.07 \mathrm{mg} / \mathrm{dL})$, and the level of Apo-B $(1.02 \pm 0.25 \mathrm{mg} / \mathrm{dL})$ 
Table 2 The levels of lipids between the ESCC patients and healthy controls

\begin{tabular}{|c|c|c|c|c|c|}
\hline & \multicolumn{2}{|l|}{ ESCC } & \multicolumn{2}{|l|}{ Normal } & \multirow[t]{2}{*}{$p$ Value } \\
\hline & Median & (range) & Median & (range) & \\
\hline ApoA-I(g/L) & 1.21 & $0.71-2.05$ & 1.56 & $0.8-2.25$ & 0.000 \\
\hline$A p o B(g / L)$ & 1.00 & $0.41-1.81$ & 0.96 & $0.41-1.88$ & 0.042 \\
\hline $\mathrm{HDL}-\mathrm{C}(\mathrm{mmol} / \mathrm{L})$ & 1.20 & $0.39-2.34$ & 1.40 & $0.68-2.47$ & 0.000 \\
\hline LDL-C(mmol/L) & 3.38 & $1.32-7.09$ & 3.42 & $1.17-6.11$ & 0.583 \\
\hline $\mathrm{TC}(\mathrm{mmol} / \mathrm{L})$ & 4.89 & $2.72-7.57$ & 5.59 & $2.57-8.92$ & 0.000 \\
\hline $\mathrm{TG}(\mathrm{mmol} / \mathrm{L})$ & 1.10 & $0.38-10.55$ & 1.12 & $0.4-12.91$ & 0.534 \\
\hline
\end{tabular}

Bold values represent the P-value $<0.05$, which was considered to be statistically significant

in ESCC patients was higher than that in healthy controls $(0.97 \pm 0.25 \mathrm{mg} / \mathrm{dL})$. However, there were no significantly differences in TG and LDL-C between ESCC patients $(1.26 \pm 0.89$ and $3.50 \pm 0.94)$ and healthy controls (1.48 \pm 1.42 and $3.42 \pm 0.98$ ) (Fig. 1).

\section{Univariate and multivariate analysis of clinicopathologic characteristics and lipids in ESCC patients}

To determine the prognostic value of pre-treatment lipids and lipoproteins in ESCC patients, the clinical characteristics (including age, gender, tobacco index, alcohol index, pT status, pN status, pM status, pTNM status, and histological differentiation) and lipid levels were subjected to univariate and multivariate analyses.
In the univariate analysis, ApoA-I [hazard ratio (HR): 1.541, $p=0.016$ ], alcohol index (Yes vs. No, HR: 0.65, $p=$ 0.015), pT status (T1-2 vs. T3-4, HR: 0.453, $p=0.001$ ), $\mathrm{pN}$ status (Yes vs. No, HR: 0.416, $p=0.000$ ), pM status (Yes vs. No, HR: $0.401, p=0.003$ ), and pTNM status (I-II vs. III-IV, HR: $0.412, p=0.000)$ were associated with significantly poor survival (Table 3 ).

To determine whether these five factors could be used as independent prognostic factors for survival, they were subjected to multivariate analysis. Considering the influence of statistical collinearity, the multivariate model did not include $\mathrm{pT}$ status, $\mathrm{pN}$ status, or $\mathrm{pM}$ status. The results showed that ApoA-I (HR: 1.519, $p=0.039$ ), alcohol index (Yes vs. No, HR: $0.688, p=0.037$ ) and pTNM status (I-II vs. III-IV, HR: $0.427, p=0.000)$ were significant independent predictors of favorable OS. Thus, our findings indicated that serum ApoA-I level before therapy may be a novel independent prognostic factor for ESCC (Fig. 2).

\section{Kaplan-Meier survival analysis of the levels of independent predictors in ESCC}

The Kaplan-Meier method was used to further explore the prognostic significance of ApoA-I level in ESCC, which was used to plot the survival curves. In the whole ESCC cohort, patients with a higher ApoA-I level showed a significantly better 5-year OS than the lower Apo-A1 group. The cumulative 5-year survival rate in the higher ApoA-I group was $48.54 \%$, whereas it was

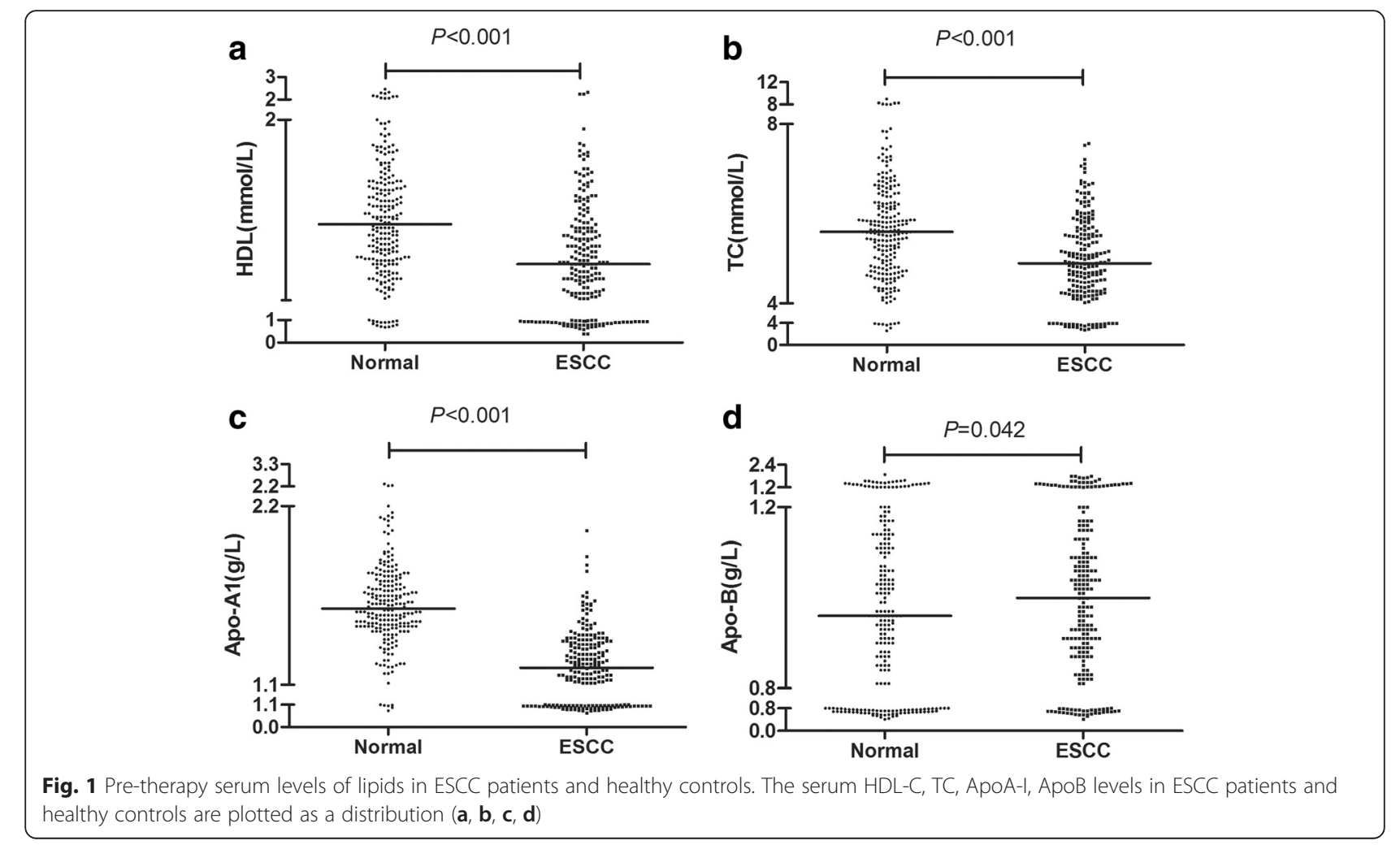


Table 3 Univariate and multivariate cox analysis for overall survival in patients with ESCC

\begin{tabular}{|c|c|c|c|c|c|c|}
\hline \multirow[t]{2}{*}{ Variables } & \multicolumn{3}{|c|}{ Univariate analysis } & \multicolumn{3}{|c|}{ Multivariate analysis } \\
\hline & $\overline{\mathrm{HR}}$ & $95 \% \mathrm{Cl}$ & $p$ Value & $\mathrm{HR}$ & $95 \% \mathrm{Cl}$ & $p$ Value \\
\hline \multicolumn{7}{|l|}{ Sex } \\
\hline Male vs. Female & 1.376 & $0.918-2.063$ & 0.123 & & & \\
\hline \multicolumn{7}{|l|}{ Age } \\
\hline$<58$ vs. $\geq 58$ & 1.008 & $0.712-1.428$ & 0.965 & & & \\
\hline \multicolumn{7}{|l|}{ pT status } \\
\hline T1-2 vs. T3-4 & 0.453 & $0.288-0.712$ & 0.001 & & & \\
\hline \multicolumn{7}{|l|}{ pN status } \\
\hline Yes vs. No & 0.416 & $0.292-0.594$ & 0.000 & & & \\
\hline \multicolumn{7}{|l|}{ pM status } \\
\hline Yes vs. No & 0.401 & $0.220-0.729$ & 0.003 & & & \\
\hline \multicolumn{7}{|l|}{ pTNM status } \\
\hline |-I| vs. |||-IV & 0.412 & $0.289-0.587$ & 0.000 & 0.427 & $0.299-0.609$ & 0.000 \\
\hline \multicolumn{7}{|l|}{ Histological differentiation } \\
\hline Differentiated vs. Undifferentiated & 0.907 & $0.227-3.722$ & 0.907 & & & \\
\hline \multicolumn{7}{|l|}{ Tobacco index } \\
\hline Yes vs. No & 0.762 & $0.528-1.101$ & 0.147 & & & \\
\hline \multicolumn{7}{|l|}{ Alcohol index } \\
\hline Yes vs. No & 0.65 & $0.458-0.921$ & 0.015 & 0.688 & $0.484-0.977$ & 0.037 \\
\hline \multicolumn{7}{|l|}{ ApoA-I (g/L) } \\
\hline$<1.21$ vs. $\geq 1.21$ & 1.541 & $1.082-2.193$ & 0.016 & 1.519 & $1.021-2.261$ & 0.039 \\
\hline \multicolumn{7}{|l|}{ ApoB(g/L) } \\
\hline$<1.00$ vs. $\geq 1.00$ & 0.927 & $0.654-1.313$ & 0.669 & & & \\
\hline \multicolumn{7}{|l|}{ HDL-C(mmol/L) } \\
\hline$<1.20$ vs. $\geq 1.20$ & 0.993 & $0.701-1.407$ & 0.968 & & & \\
\hline \multicolumn{7}{|l|}{ LDL-C(mmol/L) } \\
\hline$<3.38$ vs. $\geq 3.38$ & 0.935 & $0.659-1.327$ & 0.707 & & & \\
\hline \multicolumn{7}{|l|}{$\mathrm{TC}(\mathrm{mmol} / \mathrm{L})$} \\
\hline$<4.89$ vs. $\geq 4.89$ & 1.041 & $0.734-1.475$ & 0.824 & & & \\
\hline \multicolumn{7}{|l|}{$\mathrm{TG}(\mathrm{g} / \mathrm{L})$} \\
\hline$<1.10$ vs. $\geq 1.10$ & 1.111 & $0.784-1.574$ & 0.554 & & & \\
\hline
\end{tabular}

only $30.84 \%$ in the low ApoA-I group. Furthermore, a high alcohol index and p TNM status were associated with poor 5-year OS in ESCC patients (Fig. 2).

\section{Discussion}

The lack of accurate prognostic biomarkers for patients with ESCC limits therapeutic development, and the long-term survival of these patients remains low. Therefore, the identification of novel prognostic biomarkers for patients with ESCC is critical. In the present study, we compared the levels of serum lipids and lipoproteins (ApoA-I, Apo-B, HDL-C, LDL-C, TC and TG) between ESCC patients and controls. We found that the levels of ApoA-I, HDL-C, and TC were significantly lower, whereas Apo-B was higher in patients with ESCC than in healthy controls. In addition, OS rates were significantly poorer in patients with decreased ApoA-I levels than in patients with high ApoA-I levels, and this was independent of other variables predicting the prognosis of ESCC patients. In addition, alcohol index was an independent prognostic indicator for ESCC, and pTNM status was an independent prognostic factor for poor survival outcome. After adjustment for clinical characteristics, ApoA-I levels were not associated with age, sex, pT status, pN status, $\mathrm{pM}$ status, TNM status, histological differentiation or alcohol index.

ApoA-I, a major HDL component in serum, is synthesized in the liver and small intestine, and constitutes 

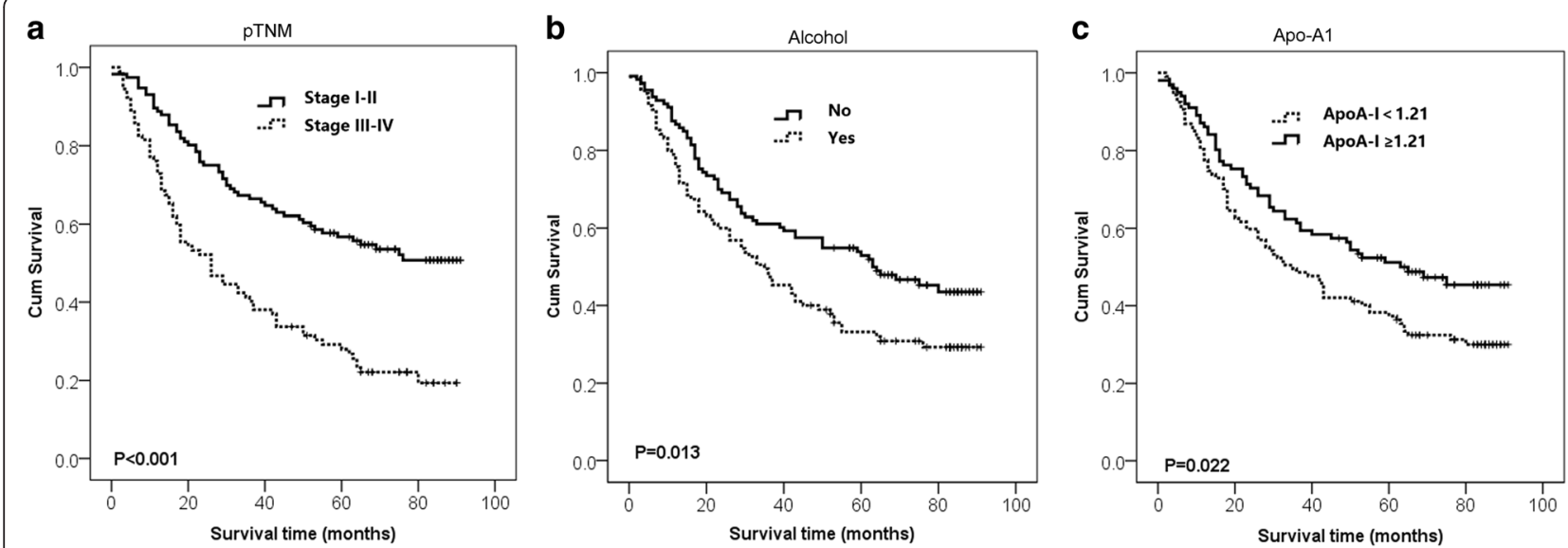

Fig. 2 Analysis of 5-year overall survival in ESCC patients. Kaplan-Meier survival curves for overall survival of ESCC patients showing significantly poor survival with higher p TNM status (a), alcohol index (b) and lower ApoA-I (c)

approximately $70 \%$ of the apolipoprotein content of HDL particles [21]. Chylomicrons contain ApoA-I, which is quickly transferred to HDL in the bloodstream after being secreted from intestinal enterocytes [22]. ApoA-I is a critical player in reverse cholesterol transport by extracting cholesterol from tissues and transferring it to the liver for its excretion. Cholesterol is a cofactor for lecithin cholesterol acyltransferase, which is responsible for the formation of most plasma cholesteryl esters [23]. ApoA-I is overexpressed in patients with recurrent head and neck squamous cell carcinoma [17]. However, ApoA-I levels are decreased in the serum of patients with pancreatic cancer, colorectal cancer, and ovarian cancer [13, 24, 25]. Low ApoA-I levels are associated with a high cancer risk, specifically with recurrence in breast cancer $[8,26]$. Similarly, our study showed that a low ApoA-I level is strongly correlated with poor OS and is an independent prognostic factor for survival (HR: 1.519; 95 \% CI: 1.021-2.261).

However, the role of ApoA-I in carcinogenesis is not well understood. In addition to the known functions of ApoA-I, it also possesses anti-inflammatory and antioxidant properties. The group of Farias-Eisner showed that ApoA-I mimetic peptides affect tumor growth and development in mouse models of colon cancer and ovarian cancer $[27,28]$. These studies can help establish an association between ApoA-I and ESCC. Lysophospholipids, such as lysophosphatidic acid (LPA), are well-known activators of proliferation in many cancers. A plausible mechanism is that ApoA-I binds LPA to inhibit LPAinduced cell growth. Hazen et al. demonstrated that in the tumor microenvironment, ApoA-I may be work as a potent immunomodulatory agent, altering tumorassociated macrophages from a pro-tumor to an antitumor phenotype [29]. Consistent with these studies, our results showed that patients with low ApoA-I levels had a significantly poor 5-year OS; however, further research is needed to clarify the underlying mechanisms.

In our study, a low alcohol index was a significant independent predictor of favorable OS in ESCC patients (HR: 0.688, 95 \% CI: 0.484-0.977). These findings are consistent with previous studies showing a strong association between alcohol consumption and increased risk of ESCC [30, 31]. There is strong epidemiological evidence that consumption of alcoholic beverages increases the risk of cancers of the oral cavity and pharynx, esophagus, and larynx. Accumulating evidence indicates that acetaldehyde is predominantly responsible for the alcohol associated carcinogenesis, since acetaldehyde is carcinogenic, mutagenic, binds to DNA and protein, destroys folate, and results in secondary hyperregeneration [32]. A number of plausible mechanisms have been proposed, including the relation between alcohol drinking and TC, HDL-C, and apolipoproteins; however, none of these has as yet been firmly established. In the present study, alcohol index was not significantly different between the two categorical ApoA-I groups (Table 1). These results indicated that alcohol consumption was not correlated with ApoA-I concentration. Further studies are needed to clarify these mechanisms.

Although ApoA-I was reported to be a prognostic factor in several malignancies [33], an association between ApoA-I and prognosis in ESCC patients has not been reported to date. In this study, the ApoA-I level was lower in ESCC patients than in normal controls, both ApoA-I and alcohol consumption are independent predictor of OS. Furthermore ApoA-I could function as an independent prognostic factor of ESCC, also it is a common and convenient monitoring index in routine preoperative examination. 


\section{Conclusions}

To our knowledge, this is the first retrospective study investigating the relationship between pre-therapy serum lipids and lipoproteins and ESCC. ApoA-I concentration is an independent favorable prognostic factor in ESCC; it shows high reproducibility and can easily be measured in all diagnostic laboratories. Further, we also demonstrated that alcohol consumption influences the survival of patients with ESCC. As this study is a retrospective analysis, it is only valid for generating a hypothesis, and the value of ApoA-I should be validated in large prospective trials.

\section{Abbreviations \\ ApoA-I, apolipoprotein A-l; Apo-B, apolipoprotein B; CEA, carcino-embryonic antigen; CYFRA21-1, cytokeratin 19 fragments; ESCC, esophageal squamous cell carcinoma; HDL-C, high-density lipoprotein-cholesterol; LDL-C, low- density lipoprotein-cholesterol; SCC, squamous cell carcinoma antigen; TC, total cholesterol; TG, triglyceride}

\section{Acknowledgments}

We thank the staff of the biochemical lab of Sun Yat-sen University Cancer Center, who provided various biochemical markers, and all the staff who supported our study.

\section{Funding}

This study was financially supported by Guangdong Esophageal Cancer Institute Project (No.Q201405)

\section{Availability of data and materials}

Due to ethical restrictions, the raw data underlying this paper are available upon request to the corresponding author.

\section{Authors' contributions}

XPW and XHL carried out the main work and contributed equally. They participated in the design of the study and drafted the manuscript. LZ, JHL and $\mathrm{HH}$ performed the statistical analysis. $\mathrm{HC}$ and $\mathrm{XZ}$ conceived the study and participated in its design and coordination, MJM and TK helped to draft the manuscript. All authors read and approved the final manuscript.

\section{Competing interests}

The authors declare that they have no competing interests. There are no financial and non-financial competing interests (political, personal, religious, ideological, academic, intellectual, commercial or any other) to declare in relation to this manuscript.

\section{Author details}

'Department of Laboratory Medicine, State Key Laboratory of Oncology in South China, Collaborative Innovation Center for Cancer Medicine, Sun Yat-sen University Cancer Center, Guangzhou, Guangdong 510060, People's Republic of China. ${ }^{2}$ Department of Laboratory Medicine, The First Affiliated Hospital of Sun Yat-sen University, Guangzhou, Guangdong, China. ${ }^{3}$ Guangdong Esophageal Cancer Institute, Guangzhou, Guangdong, China.

Received: 6 April 2015 Accepted: 4 July 2016

Published online: 21 July 2016

\section{References}

1. Enzinger PC, Mayer RJ. Esophageal cancer. N Engl J Med. 2003. 349(23):2241-52.

2. Tang WR, Chen ZJ, Lin K, Su M, Au WW. Development of esophageal cancer in Chaoshan region, China: association with environmental, genetic and cultural factors. Int J Hyg Environ Health. 2015;218(1):12-8.

3. Bedenne L, Michel P, Bouche O, Milan C, Mariette C, Conroy T, Pezet D, Roullet B, Seitz JF, Herr JP, et al. Chemoradiation followed by surgery compared with chemoradiation alone in squamous cancer of the esophagus: FFCD 9102. J Clin Oncol. 2007;25(10):1160-8.
4. Lerut $T$, Coosemans W, De Leyn P, Van Raemdonck D, Deneffe G, Decker G. Treatment of esophageal carcinoma. Chest. 1999;116(6 Suppl):463S-5.

5. Zheng X, Xing S, Liu XM, Liu W, Liu D, Chi PD, Chen H, Dai SQ, Zhong Q, Zeng MS, et al. Establishment of using serum YKL-40 and SCCA in combination for the diagnosis of patients with esophageal squamous cell carcinoma. BMC Cancer. 2014:14:490.

6. Cao HH, Zhang SY, Shen JH, Wu ZY, Wu JY, Wang SH, Li EM, Xu LY. A threeprotein signature and clinical outcome in esophageal squamous cell carcinoma. Oncotarget. 2014;6:5435-48.

7. Chen GQ, Tian H, Yue WM, Li L, Li SH, Qi L, Gao C, Si LB, Lu M. NCOA5 low expression correlates with survival in esophageal squamous cell carcinoma. Med Oncol. 2014;31(12):376.

8. Chang SJ, Hou MF, Tsai SM, Wu SH, Hou LA, Ma H, Shann TY, Wu SH, Tsai LY. The association between lipid profiles and breast cancer among Taiwanese women. Clin Chem Lab Med. 2007;45(9):1219-23.

9. Zhang X, Zhao XW, Liu DB, Han CZ, Du LL, Jing JX, Wang Y. Lipid levels in serum and cancerous tissues of colorectal cancer patients. World J Gastroenterol. 2014;20(26):8646-52

10. Cruz PM, Mo H, McConathy WJ, Sabnis N, Lacko AG. The role of cholesterol metabolism and cholesterol transport in carcinogenesis: a review of scientific findings, relevant to future cancer therapeutics. Front Pharmacol. 2013;4:119.

11. Coppola JA, Shrubsole MJ, Cai Q, Smalley WE, Dai Q, Ness RM, Fazio S, Zheng W, Murff HJ. Plasma lipid levels and colorectal adenoma risk. Cancer Causes Control. 2015;26(4):635-43.

12. Zhang Z, Bast RJ, Yu Y, Li J, Sokoll LJ, Rai AJ, Rosenzweig JM, Cameron B, Wang $Y Y$, Meng $X Y$, et al. Three biomarkers identified from serum proteomic analysis for the detection of early stage ovarian cancer. Cancer Res. 2004;64(16):5882-90.

13. Ehmann M, Felix K, Hartmann D, Schnolzer M, Nees M, Vorderwulbecke S, Bogumil R, Buchler MW, Friess H. Identification of potential markers for the detection of pancreatic cancer through comparative serum protein expression profiling. Pancreas. 2007;34(2):205-14

14. Chetty PS, Mayne L, Lund-Katz S, Stranz D, Englander SW, Phillips MC. Helical structure and stability in human apolipoprotein A-I by hydrogen exchange and mass spectrometry. Proc Natl Acad Sci U S A. 2009;106(45): 19005-10.

15. Huang HL, Stasyk T, Morandell S, Dieplinger H, Falkensammer G, Griesmacher A, Mogg M, Schreiber M, Feuerstein I, Huck CW, et al. Biomarker discovery in breast cancer serum using 2-D differential gel electrophoresis/MALDI-TOF/TOF and data validation by routine clinical assays. Electrophoresis. 2006;27(8):1641-50.

16. Sung KC, Ryu S, Wild SH, Byrne CD. An increased high-density lipoprotein cholesterol/apolipoprotein A-I ratio is associated with increased cardiovascular and all-cause mortality. Heart. 2015;101(7):553-8.

17. Gourin CG, Zhi W, Adam BL. Proteomic identification of serum biomarkers for head and neck cancer surveillance. Laryngoscope. 2009:119(7):1291-302.

18. Tung CL, Lin ST, Chou HC, Chen YW, Lin HC, Tung CL, Huang KJ, Chen YJ, Lee YR, Chan HL. Proteomics-based identification of plasma biomarkers in oral squamous cell carcinoma. J Pharm Biomed Anal. 2013;75:7-17.

19. Jiang R, Yang ZH, Luo DH, Guo L, Sun R, Chen QY, Huang PY, Qiu F, Zou X, Cao KJ, et al. Elevated apolipoprotein A-l levels are associated with favorable prognosis in metastatic nasopharyngeal carcinoma. Med Oncol. 2014;31(8):80.

20. Cao X, Li Y, Luo RZ, He LR, Yang J, Zeng MS, Wen ZS. Tyrosine-protein phosphatase nonreceptor type 12 is a novel prognostic biomarker for esophageal squamous cell carcinoma. Ann Thorac Surg. 2012;93(5):1674-80.

21. Chetty PS, Nguyen D, Nickel M, Lund-Katz S, Mayne L, Englander SW, Phillips MC. Comparison of apoA-I helical structure and stability in discoidal and spherical HDL particles by HX and mass spectrometry. J Lipid Res. 2013; 54(6):1589-97.

22. Wasan KM, Brocks DR, Lee SD, Sachs-Barrable K, Thornton SJ. Impact of lipoproteins on the biological activity and disposition of hydrophobic drugs: implications for drug discovery. Nat Rev Drug Discov. 2008;7(1):84-99.

23. Yui Y, Aoyama T, Morishita H, Takahashi M, Takatsu Y, Kawai C. Serum prostacyclin stabilizing factor is identical to apolipoprotein A-I (Apo A-I). A novel function of Apo A-I. J Clin Invest. 1988;82(3):803-7.

24. Dieplinger $H$, Ankerst DP, Burges $A$, Lenhard $M$, Lingenhel A, Fineder $L$, Buchner H, Stieber P. Afamin and apolipoprotein A-IV: novel protein markers for ovarian cancer. Cancer Epidemiol Biomarkers Prev. 2009;18(4):1127-33. 
25. Tuft SH, Nymoen DA, Hetland FT, Kaern J, Trope CG, Davidson B. APOA1 mRNA expression in ovarian serous carcinoma effusions is a marker of longer survival. Am J Clin Pathol. 2014;142(1):51-7.

26. Lane DM, Boatman KK, McConathy WJ. Serum lipids and apolipoproteins in women with breast masses. Breast Cancer Res Treat. 1995;34(2):161-9.

27. Su F, Grijalva V, Navab K, Ganapathy E, Meriwether D, Imaizumi S, Navab M, Fogelman AM, Reddy ST, Farias-Eisner R. HDL mimetics inhibit tumor development in both induced and spontaneous mouse models of colon cancer. Mol Cancer Ther. 2012;11(6):1311-9.

28. Su F, Kozak KR, Imaizumi S, Gao F, Amneus MW, Grijalva V, Ng C, Wagner A, Hough G, Farias-Eisner G, et al. Apolipoprotein A-I (apoA-l) and apoA-I mimetic peptides inhibit tumor development in a mouse model of ovarian cancer. Proc Natl Acad Sci U S A. 2010;107(46):19997-20002.

29. Zamanian-Daryoush M, Lindner D, Tallant TC, Wang Z, Buffa J, Klipfell E, Parker Y, Hatala D, Parsons-Wingerter P, Rayman P, et al. The cardioprotective protein apolipoprotein A1 promotes potent antitumorigenic effects. J Biol Chem. 2013;288(29):21237-52.

30. Bagnardi V, Blangiardo M, La Vecchia C, Corrao G. A meta-analysis of alcohol drinking and cancer risk. Br J Cancer. 2001;85(11):1700-5.

31. Corrao G, Bagnardi V, Zambon A, La Vecchia C. A meta-analysis of alcohol consumption and the risk of 15 diseases. Prev Med. 2004;38(5):613-9.

32. Doll R, Peto R. Mortality and alcohol consumption. BMJ. 1995;310(6977):470.

33. Li C, Li H, Zhang T, Li J, Liu L, Chang J. Discovery of Apo-A1 as a potential bladder cancer biomarker by urine proteomics and analysis. Biochem Biophys Res Commun. 2014;446(4):1047-52.

\section{Submit your next manuscript to BioMed Central and we will help you at every step:}

- We accept pre-submission inquiries

- Our selector tool helps you to find the most relevant journal

- We provide round the clock customer support

- Convenient online submission

- Thorough peer review

- Inclusion in PubMed and all major indexing services

- Maximum visibility for your research

Submit your manuscript at www.biomedcentral.com/submit

) Biomed Central 